\title{
Investigating Existing Medical CT Segmentation Techniques within Automated Baggage and Package Inspection
}

\author{
Najla Megherbi, Toby P. Breckon, Greg T. Flitton \\ School of Engineering, Cranfield University, Bedfordshire, UK
}

\begin{abstract}
3D Computed Tomography (CT) image segmentation is already well established tool in medical research and in routine daily clinical practice. However, such techniques have not been used in the context of $3 \mathrm{D} \mathrm{CT}$ image segmentation for baggage and package security screening using CT imagery. CT systems are increasingly used in airports for security baggage examination. We propose in this contribution an investigation of the current 3D CT medical image segmentation methods for use in this new domain. Experimental results of 3D segmentation on real CT baggage security imagery using a range of techniques are presented and discussed.
\end{abstract}

\section{INTRODUCTION}

In recent times, aviation security has received significant attention worldwide. Security screening technologies such as CT systems are becoming increasingly used for baggage examination to detect potential threats [1].

Currently screening is a manual inspection process based on human interpretation of 2D X-ray (or 3D CT density) images. This process is tedious and subject to operator's skill and training [1]. Also the image interpretation process itself is difficult task in the case of cluttered baggage with overlapping objects. This is clearly exemplified in Figure 1 which displays 2D Xray and $3 \mathrm{D} \mathrm{CT}$ images of the same cluttered baggage. In this case the detection of a threat object occluded by other objects by a simple visual inspection of the baggage imagery is difficult. It is therefore desirable to provide a functionality that allows the operator to specify objects of interest for examination and to separate them out to view them individually. Displaying and manipulating the object in 3D allows the operator to access information which is not available in a 2D X-ray image and provides better viewing and interpretation than traditional 2D inspection techniques. Such functionality can be achieved by integrating 3D object segmentation techniques into this visual inspection process. Furthermore segmentation for object isolation within manual baggage imagery interpretation is complemented by recent advances in automatic object classification within the screening process using both $3 \mathrm{D}$ CT $[2-6]$ and 2D X-ray imagery $[7,8]$.

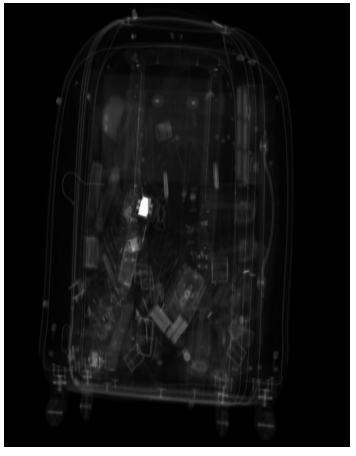

(a

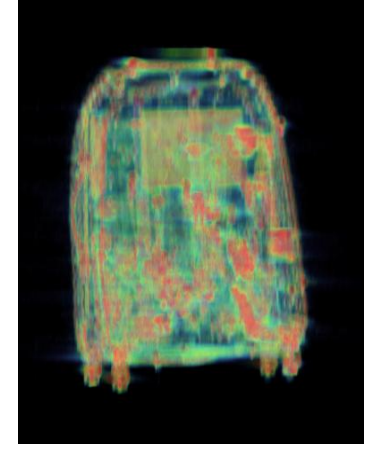

(b)
Figure 1. (a) 2D X-ray and (b) 3D CT images of the same cluttered baggage.

Such functionality has the advantage that it reduces the amount of visual data that needs to be analysed and focuses analysis on suspicious objects found in the baggage. Also it allows consistency, minimizes subjectivity and most importantly improves reliability and efficiency of the overall process [1].

From the literature, no previous work has been done on the the evaluation of medical 3D CT segmentation into the baggage inspection process [9-19] with only limited prior work addressing the security screening context but using medical quality CT imagery [20, 21]. By contrast the use of such techniques in the domain of medical imaging [22] is well established both in the field of exploratory medical research and in routine daily clinical practice. In this paper we present a novel work which aims to investigate the effective use of 3D CT medical image segmentation techniques and how they can be applied to the task of automating the existing manual CT baggage inspection processes [1] and contributing to future screening automation $[2,3,8]$.

\section{CT IMAGE SEGMENTATION OVERVIEW}

Medical image segmentation plays an important role in biomedical imaging. It has numerous applications such as study of anatomical structures, diagnosis and localization of pathology, treatment planning, and computer-guided surgery [22]. 
Classically, image segmentation is defined as the process of partitioning an image into non overlapping regions that are homogeneous according to one or several characteristics such as intensity and texture patterns. In medical imaging applications, image segmentation refers to the process of separation and delineation of an anatomical structure so that it can be viewed individually for further examination [22].

A wide variety of techniques are available for medical image segmentation [22-24]. These vary depending on the specific application, imaging modality used for gathering data (e.g. CT, MRI), and the anatomical structures reputed to be segmented. These methods can be broadly classified into two categories, namely (a) region-based methods which perform the segmentation by finding coherent regions according to some criteria [25-31], and (b) boundary-based methods that find the boundaries of the object of interest [32-40].

Region-based segmentation approaches include many variants of the region growing method [25-30] and a fuzzy connectedness segmentation method [31].

Boundary-based segmentation approaches range from the earliest and simplest threshold-based techniques $[28,41,42]$ to the more sophisticated modelbased techniques such as active contours $[32,33]$ and level sets [34-38] based on local gradients. The watershed segmentation method $[39,40]$ can also be considered under this category since it uses the boundary edge of the object of interest.

As the number of available segmentation algorithms in each category is very high, we have selected the most prominent methods to apply to our new problem domain. Notably, threshold-based techniques are not suitable in our application for two reasons. The first is the lack of homogeneity in CT density values within the same object due to object complexity. For instance an Improvised Explosive Device (IED) can have several CT density values corresponding to its different components. This is dissimilar to medical CT imagery where the anatomical structures to be segmented commonly have uniform CT density. The second reason is that objects of interest will commonly be surrounded by large number of diverse objects of different CT density value which leads to have a poor contrast against the background.

In addition as some objects, such as weapons, have regions of high curvature, level set methods using curvature term can not be readily used for this application as they are generally unsuitable for segmenting objects with high curvatures $[34,35]$. Furthermore, unlike in medical imaging applications, no prior knowledge about the shape and intensity of an object being segmented is available. As a result we exclude several methods that make use of intensity prior knowledge such those simplistic methods using an intensity range to perform the segmentation (connected threshold, neighbourhood connected, and isolated connected region growing methods) [43]. Methods based on shape prior knowledge (e.g. [36]) are also not used in this work.

Consequently, the four methods considered for evaluation in this work based on their suitability to the problem domain, are confidence connected region growing method [43] and fuzzy connectedness method [31] for region-based methods. For boundary-based approaches we have chosen the fast marching method [37] as level set method and the watershed method $[39,40]$.

\subsection{Confidence Connected Region Growing}

Region growing segmentation techniques start the segmentation by defining a seed region (one or more voxels) placed inside the object of interest. Neighbouring voxels are then evaluated against a predefined homogeneity criterion, e.g. intensity, color, and texture. Voxels that satisfy the criterion are added to the region. This process is repeated until the whole volume is covered leading the region to grow from the given seed region. The resulting connected voxels in the volume represent the object of interest. The confidence connected method is a simplest implementation of region growing segmentation approaches in which the homogeneity criterion is a function of CT density values statistics (e.g. mean and standard deviation) of the object of interest [43].

\subsection{Fuzzy Connectedness}

As in the previous segmentation method, this method looks for uniformity within a sub-region, based on a desired property. The later is represented by a fuzzy affinity measure which computes the strength of connectedness between any pair of voxels in the volume [31]. This measure is a function of the degree of the adjacency of the considered voxels and the similarity of their CT density values. The greater the spatial proximity and the similarity CT density, the greater the affinity map between given voxels [31].

\subsection{Watershed}

The watershed method is based on the topography concept of watersheds and catchment basins [39, 40]. If we flood a surface with waters form minimal altitudes (minima) such that we avoid the merging of waters coming from the different minima, the surface will be separated into catchment basins and watershed lines. By 
analogy to this process, the gradient image of the CT volume is interpreted as a topographic map where the CT levels at each point describe altitudes. Catchment basins correspond to the homogeneous sub-regions in the volume and the watershed lines correspond to the edges. The key advantage of watershed method is that it is automatic where no seed points are needed to perform the segmentation. In addition it always produces closed contours. This method is notably very sensitive to noise which can result in over-segmentation of the volume.

\subsection{Fast Marching}

The Fast Marching method works by propagating a front from an initial seed region. The evolution of this front results from applying a general speed function to the initial seed region. Usually the speed function is an edge-attracting function which is constructed so that it moves quickly in homogeneous areas when the gradient is low and stops in region edge when the gradient is high. The Fast Marching method is a type of level set method which is designed for problems in which the sign of the speed function is preserved (always positive or negative) so that the front always moves outward or inward. Since the speed function never changes sign, the location of the propagating front, hence the boundary of the region to be segmented is computed as the arrival time of the front as it crosses the voxels in the volume. This time is a function of the distance from the initial seed region and the speed function which represent the resistance against the front as it progresses through the volume [37].

\section{EXPERIMENTS AND RESULTS}

In this section we present the results of the four previous approaches for 3D CT segmentation on baggage security CT imagery. The data was gathered using a Reveal CT-80 baggage scanner with voxel resolution $1.5 \mathrm{~mm} \times 1.6 \mathrm{~mm} \times 16 \mathrm{~mm}(\mathrm{x}, \mathrm{y}, \mathrm{z})$.

Figure 2 displays two samples of our data. In the top, the 2D X-ray images are presented. In the bottom, we show the rendered volumes of the same two samples. The first sample (Figure 2 left) is a baggage which contains among other objects a bottle of water. The other sample has a gun placed in the baggage containing other different objects. In the following discussion, we will refer to these two samples as bottle baggage and gun baggage respectively.

In our experiment we are interested to segment the bottle and the gun as examples of simple and complex
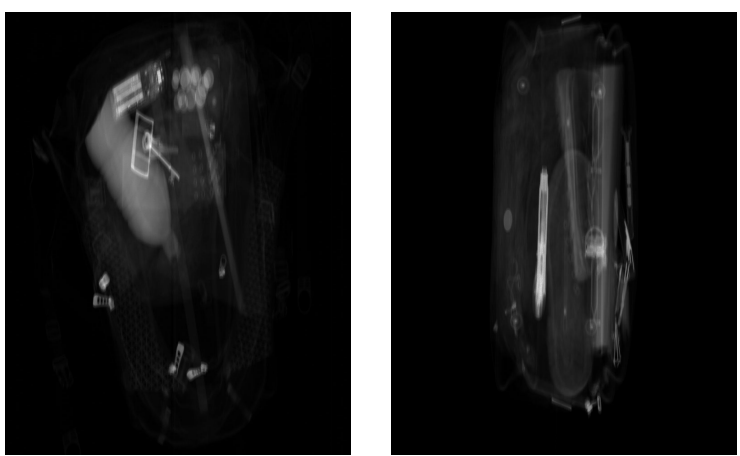

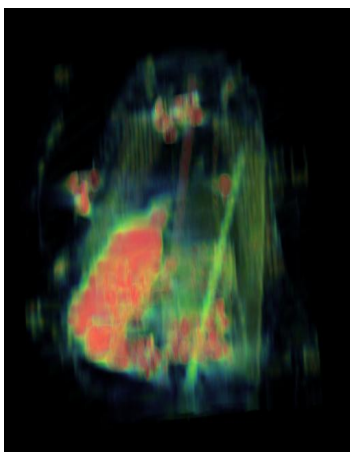

(a)

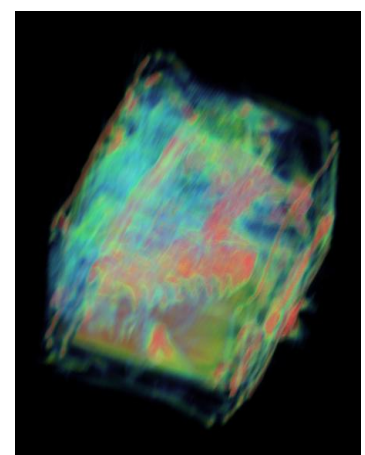

(b)
Figure 2. 2D X-ray image and CT volume of (a) bottle baggage and (b) gun baggage.

items of interest. Within current security concerns, bottles represent interesting target objects to examine in any baggage screening process.

Before applying any of the selected segmentation methods, the baggage security CT volumes are preprocessed to filter out basic image noise. To this end, a non-linear anisotropic diffusion smoothing filter is used [44]. This filter has the advantage of both preserving the edge structures and smoothing noise within the CT volume. The imagery suffers from a range of noise sources, notably caused by metal artefacts in the CT process which are themselves the subject of separate investigation [45-48].

In Figure 3 we show the results of applying confidence connected, fuzzy connectedness and fast marching methods on the bottle baggage. In Figure 4 we present different watershed segmentation results generated by varying the threshold and the water level parameters. The meaning of these parameters can be found in [43]. As the ground truth segmentation for baggage security imagery is difficult to obtain, the four methods are compared qualitatively with respect to their subjective results. This is a valid measure of evaluation based on the fact that human operators will ultimately view such imagery. 
As can be seen from Figure 3 and 4 all methods lead to good segmentation results. The bottle is segmented and separated successfully with confidence connectedness, fuzzy connectedness and fast marching methods. By analysing the slices of the segmented bottle, as depicted in Figure 5, one can remark that the region extracted with the confidence connected method has holes inside (Figure 5a). This is a common behaviour of region growing segmentation methods. In some situations the extracted regions are disconnected. The region extracted with the fuzzy connectedness is smooth compared to the previous segmentation, as can be seen in the border of the extracted region, and has fewer holes (Figure 5b). The fast marching method produces, as by definition, a connected region (Figure 5c).

The results obtained with the watershed method depend on the parameter tuning, image details (amount of edges) and the amount of noise present in the volume (Figure 4). Unlike the other methods, the watershed method operates on the whole volume and produces a total partition of the imagery which results in segmenting all objects contained in the volume. Tuning the parameters of this method to produce a segmentation of all objects without leading to an over-segmentation or merging together close regions is a difficult and tedious task.

The segmentation results of these methods can be improved by applying a post-processing step. For instance, in the case of confidence connected segmentation(Figure 3a), a morphology dilation filter can be used to close the holes inside the segmented object.

In Figure 6 we show the results of the gun segmentation using the four methods. The confidence connected and the fuzzy connectedness methods perform well (Figure 6 a,b). With few seed points, the gun has been segmented and the same remarks as for the results of these two methods on the bottle hold for this example also. As for the bottle, the results of applying the watershed method also depend on parameters tuning and image details (Figure 6d).

As Figure $6 \mathrm{c}$ reveals, the fast marching method failed on the gun. This is due to the fact that there is not enough area where the required marching front can evolve from the initial seed points toward the edges of the gun. This problem is caused by two factors: the size of the edges which is comparable to the size of the gun (as depicted in Figure 7), and the slice thickness (spacing between adjacent $\mathrm{CT}$ slices) in the $\mathrm{z}$ direction: In our work, the CT scanner used has a z slice thickness of $16 \mathrm{~mm}$ compared to $1.5 \mathrm{~mm}$ (or finer) in medical applications. Such resolution prevents us segmenting small

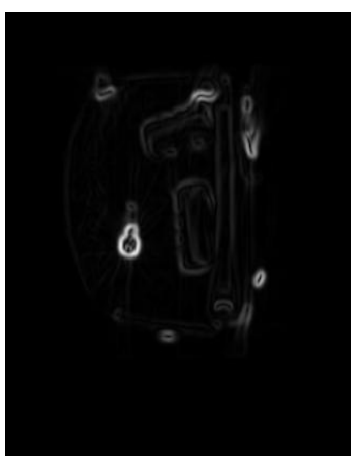

Figure 7. An axial slice of the gun baggage gradient image.

and thin objects such as batteries and knifes.

\section{CONCLUSION}

In this contribution, we attempt to answer the question how suitable are current medical based 3D CT segmentation techniques for application to baggage security CT imagery. Experiments show that the current 3D CT medical image segmentation methods can be successfully applied to the CT security screening problem domain but that the results are significantly limited by the presence of noise, the complexity of the target imagery within this context and the lack of prior domain knowledge that underpins a range of leading medical domain approaches. In particular, we conclude that region-based segmentation methods perform better than boundary-based region methods especially for small objects.

The problem domain chosen is challenging compared to medical imaging applications. In our experiment, the parameters of the segmentation methods have to be tuned for each object we are interested in segmenting and also for each baggage item. In other words, a set of parameters working for an object placed in a given baggage item may not work for the same object placed in another baggage item. This is because the object will be surrounded by different contextual objects each time it is placed in a different bag. In medical applications, such problem does not occur since the location (the background) of the anatomical structure to be segmented is known a priori. In addition, no prior knowledge about the shape and intensity is available unlike medical equivalent. Furthermore, threat objects lack for CT density homogeneity unlike anatomical structures in medical applications.

Overall, the application of medical CT segmentation to CT security screening shows promise but key differences in the subject of the imagery results in new challenges unforeseen in prior medical work. Our evaluation 


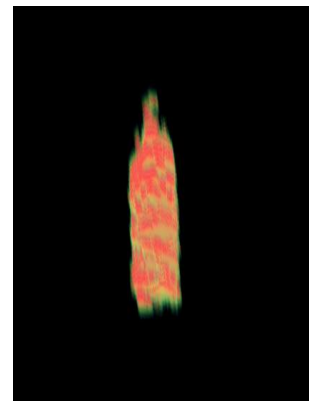

(a)

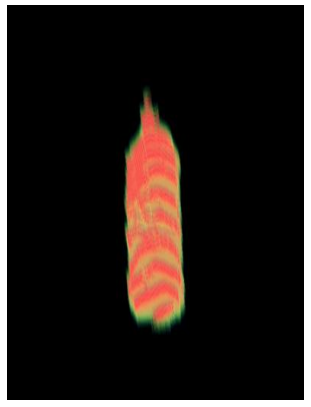

(b)

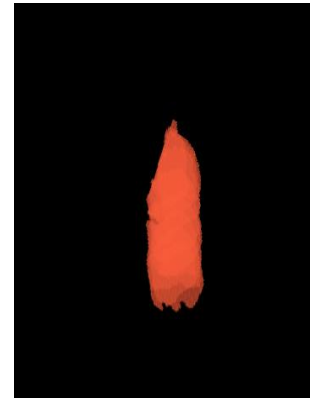

(c)

Figure 3. 3D bottle segmentation: (a) confidence connected, (b) fuzzy connectedness, (c) fast marching
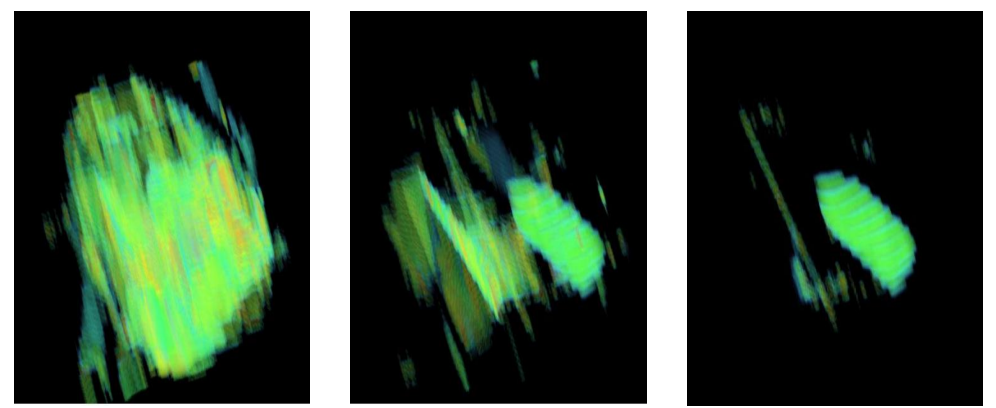

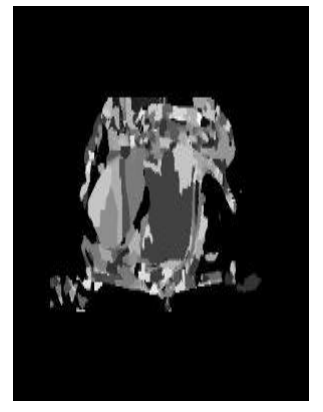

(a)

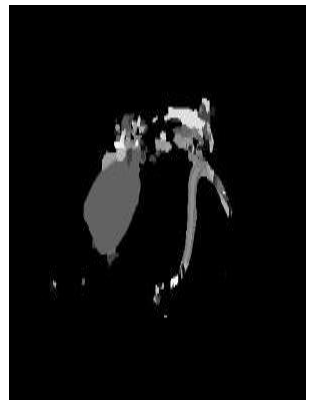

(b)

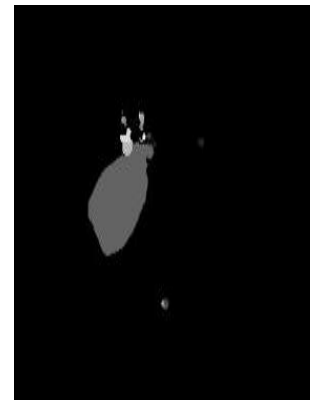

(c)

Figure 4. 3D watershed segmentation of the bottle for various parameters (a) threshold $=0.001$ and water level $=0.05$ (b) threshold $=0.01$ and water level $=0.05$, (c) threshold $=0.01$ and water level $=0.15$. Top are the rendered volumes, bottom are axial slices.

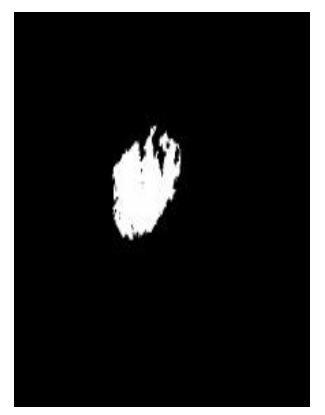

(a)

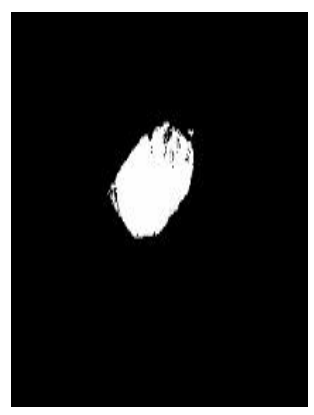

(b)

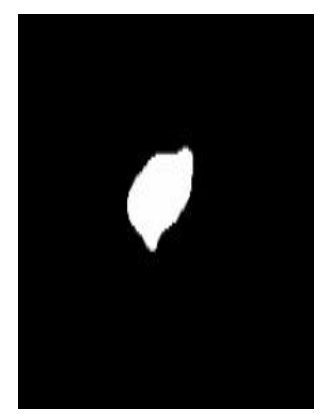

(c)

Figure 5. Axial slices of the segmented bottle using: (a) confidence connected (b) fuzzy conectdness and (c) fast marching methods. 


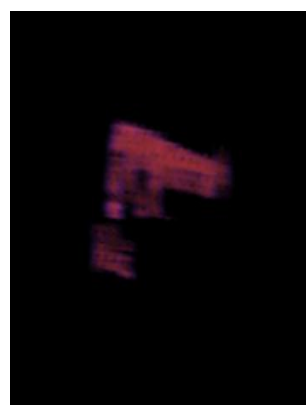

(a)

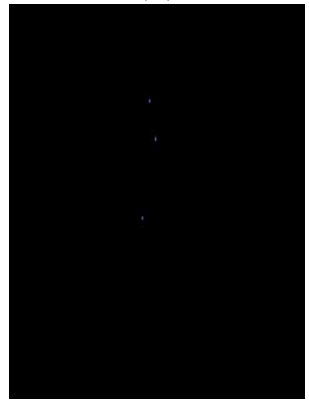

(c)

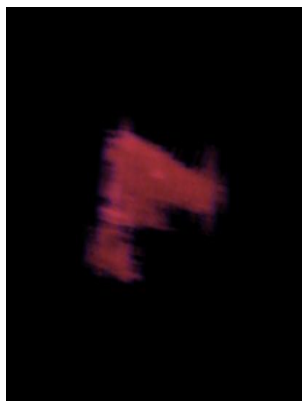

(b)

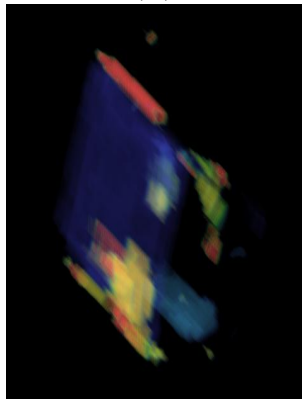

(d)

Figure 6. 3D gun segmentation: (a) confidence connected, (b) fuzzy connectedness, (c) fast marching, (d) watershed.

here will form the basis for future work in automated object extraction for automated threat/non threat classification for security screening $[2,4-6]$.

This project was funded under the Innovative Research Call in Explosives and Weapons Detection 2007 \& 2010 initiative. This is a Cross-Government programme sponsored by a number of Departments and Agencies under the UK Government's CONTEST strategy in partnership with the US Department of Homeland Security.

\section{REFERENCES}

1. N. Shanks and A. Bradley, Handbook of Checked Baggage Screening: Advanced Airport Security Operation. WileyBlackwell, 2004.

2. N. Megherbi, J. Han, G. Flitton, and T. Breckon, "A comparison of classification approaches for threat detection in CT based baggage screening," in Proc. International Conference on Image Processing, pp. 3109-3112, IEEE, September 2012.

3. N. Megherbi, G. Flitton, and T. Breckon, "A classifier based approach for the detection of potential threats in CT based baggage screening," in Proc. International Conference on Image Processing, pp. 1833-1836, IEEE, September 2010.

4. G. Flitton, T. Breckon, and N. Megherbi, "A comparison of 3D interest point descriptors with application to airport baggage object detection in com- plex CT imagery," Pattern Recognition, vol. 46, pp. 2420-2436, September 2013.

5. G. Flitton, T. Breckon, and N. Megherbi, "Object recognition using 3D SIFT in complex CT volumes," in Proc. British Machine Vision Conference, pp. 11.1-12, September 2010.

6. G. Flitton, T. Breckon, and N. Megherbi, "A 3D extension to cortex like mechanisms for 3D object class recognition," in Proc. International Conference on Computer Vision and Pattern Recognition, pp. 3634-3641, IEEE, June 2012.

7. M. Bastan, M. R. Yousefi, and T. M. Breuel, "Visual words on baggage X-ray images," in Proc. Int. Conf. on Computer Analysis of Images and Patterns, pp. 360-368, 2011.

8. D. Turcsany, A. Mouton, and T. Breckon, "Improving feature-based object recognition for x-ray baggage security screening using primed visual words," in Proc. International Conference on Industrial Technology, pp. 1140-1145, IEEE, February 2013.

9. S. Singh and M. Singh, "Explosives detection systems (EDS) for aviation security," Signal Processing, vol. 83, no. 1, pp. 31-55, 2003.

10. R. Paranjape, M. Sluser, and K. Runtz, "Segmentation of handguns in dual energy X-ray imagery of passenger carry-on-luggage," in Proceedings of the 
11th Canadian Conference on Electrical and Computer Engineering, Toronto, Canada, pp. 377-380, 1998.

11. P. Bjorkholm and T. Wang, "Contraband detection using X-rays with computer assisted image analysis," in Proceedings of the Symposium on Contraband and Cargo Inspection Technology, pp. 111115, 1992.

12. Z. Ying, R. Naidu, K. Guilbert, D. Schafer, and C. Crawford, "Dual energy volumetric X-ray tomographic sensor for luggage screening," in Proceedings of IEEE Sensors Applications Symposium, San Diego, California USA, pp. 1-6, 2007.

13. N. Murray, R. Lacey, and P. Mason, "Exploitation of X-ray technology for the detection of contraband-aviation security applications," in Proceedings of European Conference on Security and Detection, IEE, London, pp. 13-18, 1997.

14. M. Sluser and R. Paranjape, "Model-based probabilistic relaxation segmentation applied to threat detection in airport X-ray imagery," in Proceedings of the 1999 IEEE Canadian Conference on Electrical and Computer Engineering, pp. 720-726, 1999.

15. M. Singh and S. Singh, "Image segmentation optimisation for X-ray images of airline luggage," in Proceedings of IEEE International Conference on Computational Intelligence for Homeland Security and Personal Safety, Venice, Italy, pp. 10-17, 2004.

16. R. Benjamin and S. Prakoonwit, "Automatic X-ray screening of aircraft hold luggage," in Proceedings of European Conference on Security and Detection, pp. 5-9, 1997.

17. L. Jimin, B. Abidi, and M. Abidi, "Automatic Xray image segmentation for threat detection," in Proceedings of Fifth International Conference on Computational Intelligence and Multimedia Applications, pp. 396-401, 2003.

18. R. Gesick, C. Saritac, and C. Hung, "Automatic image analysis process for the detection of concealed weapons," in Proceedings of the 5th Annual Workshop on Cyber Security and Information Intelligence Research: Cyber Security and Information Intelligence Challenges and Strategies, 2009.

19. B. Abidi, Z. Yue, A. Gribok, and M. Abidi, "Improving weapon detection in single energy X-ray images through pseudo coloring," IEEE Transactions on Systems, Man, and Cybernetics, Part C: Applications and Reviews, vol. 36, no. 6, pp. 784796, 2006.
20. L. Grady, V. Singh, T. Kohlberger, C. Alvino, and C. Bahlmann, Automatic segmentation of unknown objects, with application to baggage security, pp. 430-444. European Conference on Computer Vision, Springer, 2012.

21. D. F. Wiley, D. Ghosh, and C. Woodhouse, "Automatic segmentation of ct scans of checked baggage," in Proc. of the 2nd Inter. Meeting on Image Formation in X-ray CT, pp. 310-313, 2012.

22. O. Wirjadi, "Survey of 3D image segmentation methods," 2007. Technical report No. 123, Fraunhofer ITWM, Kaiserslautern, Germany.

23. S. Lakare, "3D segmentation techniques for medical volumes," 2000. Research Proficiency, Department of Computer Science State University of New York at Stony Brook.

24. D. Pham, C. Xu, and J. Prince, "Current methods in medical image segmentation," Annual Review of Biomedical Engineering, vol. 2, pp. 315-338, 2000.

25. R. Adams and L. Bischof, "Seeded region growing," IEEE Trans. Pattern Analysis Machine Intelligence, vol. 16, no. 6, pp. 641-647, 1994.

26. C. Revol and M. Jourlin, "A new minimum variance region growing algorithm for image segmentation," Pattern Recognition Letters, vol. 18, pp. 249-258, 1997.

27. Z. Lin, T. Chen, J. Jesse, and H. Talbot, "Unseeded region growing for 3D image segmentation," in Pan-Sydney Workshop on Visual Information Processing, pp. 31-37, 2000.

28. N. Nikolaidis and I. Pitas, 3-D Image Processing Algorithms. John Wiley and Sons Inc., 2001.

29. C. Revol-Muller, F. Peyrin, Y. Carrillon, and C. Odet, "Automated 3D region growing algorithm based on an assessment function," Pattern Recognition Letters, vol. 23, pp. 137-150, 2002.

30. M. Mancas, B. Gosselin, and B. Macq, "Segmentation using a region growing thresholding image processing: algorithms and systems," in Conference No4, San Jose CA, ETATS-UNIS, vol. 5672, pp. 388-398, 2005.

31. J. Udupa and S. Samaresekera, "Fuzzy connectedness and object definition: Theory, algorithms and applications in image segmentation," Graphical Models and Image Processing, vol. 58, no. 3, pp. 246-261, 1996.

32. T. McInerney and D. Terzopoulos, "Deformable models in medical image analysis: A survey," Med. Image Anal., vol. 1, no. 2, pp. 91-108, 1996. 
33. P. Etyngier, F. Segonne, and R. Keriven, "Activecontour-based image segmentation using machine learning techniques," in Medical Image Computing and Computer-Assisted Intervention, Volume 4791, pp. 891-899, 2007.

34. R. Malladi, J. Sethian, and B. Vermuri, "Shape modeling with front propagation: A level set approach," IEEE Trans. on Pattern Analysis andMachine Intelligence, vol. 17, no. 2, pp. 158-174, 1995.

35. V. Caselles, R. Kimmel, and G. Sapiro, "Geodesic active contours," International Journal on Computer Vision, vol. 22, no. 1, pp. 61-97, 1997.

36. M. Leventon, W. Grimson, and O. Faugeras, "Statistical shape influence in geodesic active contours," in Proc. IEEE Conference on Computer Vision and Pattern Recognition, volume 1, pp. 316$323,2000$.

37. J. Sethian, Level Set Methods and Fast Marching Methods. Cambridge University Press, second ed., 1999.

38. F. Zhu and J. Tian, "Modified fast marching and level set method for medical image segmentation," journal of X-Ray Science and Technology, vol. 11, pp. 193-204, 2003.

39. P. Soille, "Morphological image analysis," 1999. Springer-Verlag.

40. J. Serra, Image Analysis and Mathematical Morphology. Academic Press Inc., 1982.

41. P. Sahoo, S. Soltani, A. Wang, and Y. Chen, "A survey of thresholding techniques," Computer Vision, Graphics, and Image Processing, vol. 41, pp. 233-260, 1988.

42. S. Lee, S. Chung, and R. Park, "A comparative performance study of several global thresholding techniques for segmentation," Computer Vision, Graphics, and Image Processing, vol. 52, pp. 171190, 1990.

43. L. Ibanez, W. Schroeder, L. Ng, and J. Cates, The ITK Software Guide: The Insight Segmentation and Registration Toolkit. Kitware Inc., 2005.

44. P. Perona and J. Malik, "Scale-space and edge detection using anisotropic diffusion," IEEE Transactions on Pattern Analysis Machine Intelligence, vol. 12, pp. 629-639, 1990.

45. A. Mouton, G. Flitton, S. Bizot, N. Megherbi, and T. Breckon, "An evaluation of CT image denoising techniques applied to baggage imagery screening," in Proc. International Conference on Industrial Technology, pp. 1063-1068, IEEE, February 2013.
46. A. Mouton, N. Megherbi, G. Flitton, S. Bizot, and T. Breckon, "A novel intensity limiting approach to metal artefact reduction in 3D CT baggage imagery," in Proc. International Conference on Image Processing, pp. 2057-2060, IEEE, September 2012.

47. A. Mouton, N. Megherbi, T. Breckon, K. Van Slambrouck, and J. Nuyts, "A distance weighted method for metal artefact reduction in CT," in Proc. International Conference on Image Processing, IEEE, September 2013. to appear.

48. A. Mouton, N. Megherbi, K. Van Slambrouck, J. Nuyts, and T. Breckon, "An experimental survey of metal artefact reduction in computed tomography," Journal of X-Ray Science and Technology, vol. 21, pp. 193-226, May 2013. 